\title{
Research of "double desserts" seismic prediction method for tight sandstone - Taking Linxing block on the east edge of Ordos Basin as an example
}

\author{
Zhang junlin ${ }^{1, *}$, Wan huan ${ }^{1}$, Liu yumin ${ }^{1}$, He yumei ${ }^{1}$, Zhang hao ${ }^{1}$ and Peng shengqin ${ }^{1}$ \\ ${ }^{1}$ CNOOC EnerTech-Drilling\&Production Co. , Tianjin 300452, China
}

\begin{abstract}
The determination of the sweet spot of tight sandstone reservoir is the primary problem in the exploration and development of tight sandstone reservoir. Practice has proved that the prediction of reservoir spatial pore characteristics, thickness distribution and oil and gas potential (i.e. geological sweet spot) of tight sandstone reservoir can not meet the demand of exploration and development of tight sandstone reservoir. In view of the requirements of tight sandstone fracturing engineering, it is also necessary to analyze the engineering field of the reservoir. In this paper, based on seismic data preprocessing and petrophysical analysis, thin reservoir quantitative characterization and AVO fluid prediction are used to evaluate the tight reservoir. At the same time, combining with the fracture analysis method of FMI imaging logging, pre stack fracture description and brittleness prediction, the "engineering sweet spot" prediction is carried out. Finally, the multi-attribute RGB digital fusion visualization research method is used to comprehensively evaluate the tight reservoir. The practice shows that the research method has guiding significance for the exploration and development of tight reservoir.
\end{abstract}

\section{Introduction}

The research on tight sandstone reservoirs started from the beginning of the 1980s. Walls (1982) was the first one who proposed the related concept of oil and gas reservoir in tight sandstone. Law (1993) defined the concept of right sandstone reservoir more clearly and pointed out the difference between tight sandstone reservoir and tight sandstone gas reservoir in the aspect of spatial occurrence position, thus guiding the prediction studies of "desserts" in tight sandstone reservoir in a certain sense. Surdam (1994) put forward the concept of tight gas "sweet spot prediction and indicated that the part with relatively good physical properties in the tight reservoir was called "sweet zone". Tong X G et al. (2012) compared the generality and difference between Chinese and American tight gas reservoirs, and further expounded the particularity of tight gas researches in China; In recent years, geophysicists from various countries have done a lot of work of sweet spot determination in tight sandstone reservoirs, and the main geophysical technologies used include seismic discontinuous analysis technology, prestack and post-stack inversion, quantitative reservoir characterization and fracture prediction technology, Amplitude Variation with Offset (AVO) fluid prediction technology, etc. Zhu X M et al. (2018) pointed out that the sweet spot formation mechanism in tight reservoirs and sweet spot determination were still the research hotspots and difficulties in the field of geoscience, and meanwhile, the scholars reflected upon the effect of natural fractures in tight sandstone reservoir on hydrofracturing in sweet zone; With further deepening of the exploration and development researches on tight sandstone reservoirs, the engineering development factors of tight sandstone reservoirs have been investigated while "geological sweet spots" in tight reservoirs are predicted. The "double sweet spots prediction", which integrates reservoir physical attribute and gas-bearing attribute-dominated "geological sweet spot" and reservoir fracture-dominated "engineering sweet spot", has become a new research hotspot regarding tight sandstone reservoirs. At present, the domestic (China) researches on tight sandstone reservoirs via this technology have been scarcely reported.

\section{Overview of research thought and method}

A tight sandstone reservoir is featured by tight gas, diversified longitudinal lithological associations and thin reservoir. In this paper, the inversion and prediction methods for tight reservoir were expounded from aspects like reservoir prediction method, effective reservoir recognition and inversion \& adaptability analysis, demonstration and analysis of the accuracy of effective reservoir prediction and related project experience, followed by an analysis of research thought and technical means.

\footnotetext{
Zhang Junlin: zhangj163@cnooc.com.cn.
} 


\subsection{Determination of geological sweet spots affiliations}

The reservoir fluid prediction based on rock geophysical analysis can relieve the blindness of reservoir fluid prediction. Relying upon the petrophysical analysis technique, this prediction method not only plays a guiding role in the reservoir fluid prediction but also provides a technical support for explaining the petrophysical attribute data sensitive to reservoir, which is acquired through inversion. As seismic attribute researches are transformed from post-stack into pre-stack researches, it is especially important to formulate the petrophysical analysis-dominated reservoir fluid prediction process. On this basis, the pre-stack and poststack combined inversion method was used to acquire petrophysical properties sensitive to reservoir. The reservoir thickness and effective reservoir thickness were predicted using lithofacies fluid probability analysis method, and meanwhile, the predicted effective reservoir thickness was verified via AVO forward fluid modeling and analysis, the success rate of effective reservoir prediction was analyzed, and the reservoir prediction results were optimized with multiple attribute data.

\subsection{Determination of engineering sweet spots}

The prediction of engineering sweet spots is of great realistic significance in the tight gas exploration and development process, including the prediction of rock mechanical properties, rock brittleness, stress and fracture development zone, etc.; The effective exploration of tight oil and gas reservoirs is faced with enormous challenges due to strong heterogeneity, low porosity, low permeability and large airflow resistance. The brittleness research has played a significant role in drilling engineering and deep rock mass fracture engineering. In recent years, brittleness has become an important parameter guiding the fracturing construction of oilfields and been applied to fracturing evaluation of unconventional reservoirs, and it is deemed that rocks with high brittleness index can be fractured more easily; The pressure of oil and gas reservoir is a reflection of oil and gas energy, and the force driving oil and gas flow in gas reservoir (oil reservoir). An accurate prediction of formation pressure helps to identify and discover new oil and gas reservoirs, understand underground oil and gas energy, control the pressure change in oil and gas reservoirs, reasonably utilize the maximum energy in oil and gas reservoirs and conduct oil and gas exploitation more efficiently.

\subsection{Visualized fusion of double sweet spots}

In view of the particularity of thin tight sandstone reservoirs, it is difficult to effectively describe their spatial distribution with few seismic attribute data, so it is necessary to comprehensively depict the thin tight sandstone reservoirs by making full use of dynamic and geometric seismic properties, and the fusion method for data of different attributes becomes particularly significant. Mass attribute data, which effectively respond to geological and engineering sweet spots in the reservoir, can be acquired based on petrophysical analysis and comprehensive and multi-dimensional seismic attribute analysis method, but a single data body may lay particular emphasis on effective description of local specific physical characteristics in the reservoir. When it comes to feasibility demonstration of an unconventional tight sandstone reservoir, the oil and gas enrichment region in the reservoir should be firstly determined, followed by fracturing argumentation of the enrichment region. The double sweet-spot prediction method based on fracturing evaluation of the enrichment region is an anchor point for predicting thin tight reservoir; Red, Green and Blue (RGB) color modelbased visualized fusion method is one of effective multiattribute fusion methods emerging in recent years. This method can reserve the original features of attribute data while implementing data fusion. The RGB color modelbased visualized seismic attribute fusion method is used to fuse double sweet spots data. Through the adjustment of fusion parameters, key parameters such as oil and gas possibility of the reservoir, advantaged fracture zone of natural fracture, and brittleness factor can be displayed transparently in a fused way, the double sweet-spot attributes of the thin tight reservoir can be displayed through fusion under the visualization platform, so as to assist in decision-making process during the tight reservoir exploration and development.

\section{Application example}

\subsection{Basic geological features of the study area}

Plate marginal tectonic activities of different natures in multiple periods have formed an overall large-scale monoclinal structure, which presents westward and NW (northwest)-trending gentle dip at the east margin of Ordos Basin, and this belongs to a transitional basin marginal tectonic region. From top to bottom, the study area is developed with the following sedimentary formations: Quaternary system, Triassic system, Permian system, Carboniferous system, Ordovician system and Cambrian system, where the Triassic formations include Yanchang Formation, Zhifang Formation, Heshanggou Formation and Liujiagou Formation; The upper Permian and middle Permian formations are Shiqianfeng Formation (P3q) and Shihezi Formation (P2h), respectively, the lower Permian formations include Shanxi Formation (P1s) and Taiyuan Formation (P1t), the upper Carboniferous formation is Benxi Formation (C2b), and the basement is middle Ordovician formation-Majiagou Formation $(\mathrm{O} 2 \mathrm{~m})$.

\subsection{Prediction of geological sweet spots}

\subsubsection{Petrophysical analysis}

Based on an analysis of the petrophysical interpretation chart, it is deemed that in this work area, mudstone and 
sandstone cannot be differentiated via P-wave impedance, and similarly, it is difficult to differentiate gas-containing sandstone from mudstone and gascontaining sandstone from water-containing sandstone through P-wave impedance. However, it can be known from the interpretation chart that velocity of Pwave/velocity of S-wave (VP/VS) ratio can be used to differentiate gas-containing sandstone from watercontaining sandstone, dry sandstone and mudstone very well. The VP/VS ratio of several rock types in the work area, e.g. dry coal sandstone, mudstone, dry sandstone and gas-containing sandstone, on the petrophysical interpretation chart has a certain elastic parameter identification window. The VP/VS ratio has favorable ability to differentiate tight sandstones in the study area, e.g. differentiating porous sand from dry sand. Through the pre-stack inversion method, the $1 \mathrm{VP} / \mathrm{VS}$ ratios of the same dimension can be acquired. The reservoirs and effective reservoirs in the study area can be effectively described (Fig. 1) by combining the response characteristics of gas-containing sandstone and dry sandstone to VP/VS ratio according to its spatial distribution characteristic.

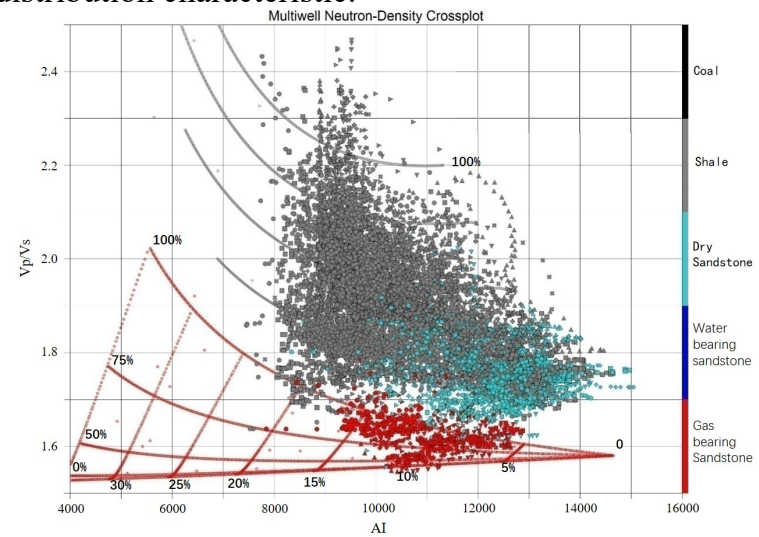

Fig. 1. Petrophysical interpretation of upper Shihezi Formation.

\subsubsection{Pre-stack gather preprocessing}

The pre-stack gather, which is the most important input data during the pre-stack inversion process, should be converted into an angle gather. On this basis, pre-stack gather will be preprocessed, such as automatic residual normal moveout (NMO) correction, wavelet reversedrawing and median filtering, and during this process, the conversion of offset gather into angle gather is the primary problem needing to be solved. By changing the background velocity and observing the change of incident angle, the change of incident angle is not stable at root mean square velocity, but it is relatively reasonable at interval velocity. Hence, the background velocity used in this inversion is determined as interval velocity (Fig. 2).

The response of tight heterogenous reservoir to seismic wave is anisotropic. Gather unevenness phenomenon will usually take place at distant offset position due to residual velocity factor in NMO correction. Through the automatic residual velocity analysis, the gather is further calibrated to eliminate the influence of residual velocity so that the gather at distant offset is leveled, and moreover, the Amplitude Variation with Offset (AVO) illusion caused by residual velocity can be eliminated (Fig. 3).

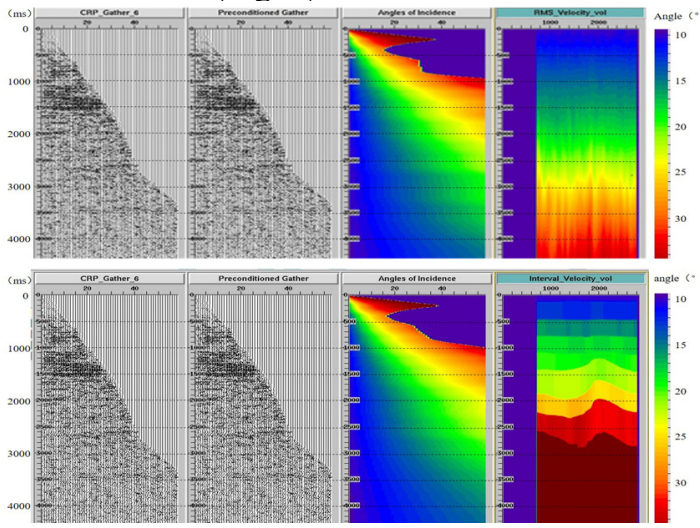

Fig. 2. Optimization of gather conversion velocity model.

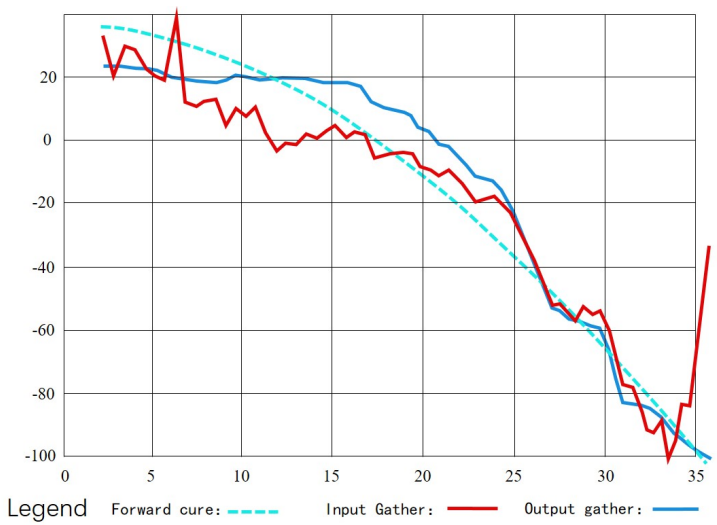

Fig. 3. Comparison chart of AVO response curves before and after gather preprocessing.

\subsubsection{Reservoir inversion}

Based on petrophysical analysis and analysis of petrophysical parameters sensitive to the reservoir, the inversion process was reasonably formulated, the petrophysical attribute data sensitive to the reservoir were acquired, a preliminary description of spatial reservoir distribution characteristics was carried out, and meanwhile, reliable input data were provided to geostatistics, and the reservoir thickness and effective thickness were predicted.

\subsection{Prediction of engineering sweet spots}

The effective exploitation of tight oil and gas reservoirs is faced with enormous challenges due to their strong heterogeneity, low porosity, low permeability and large airflow resistance. The brittleness research has exerted a great effect in drilling engineering and deep rock mass fracturing process. In recent years, brittleness has become an important parameter guiding fracturing construction of oilfields and been applied to fracturing evaluation of unconventional reservoirs, and it is deemed that rocks with high brittleness index can be fractured more easily. From Fig. 4, the elastic parameters and brittleness indexes of coal seam, mudstone and sandstone in the study area present evident zoning 
characteristics, the corresponding Poisson's ratio of sandstone is low with high brittleness index, and the corresponding brittleness of coal seam is the minimum while that of mudstone is intermediate. It can be seen from logging data and core sections that fractures are quite developed in the sandstone interval; From the angle of reservoir space, the reservoir space of tight gas reservoir is a typical multiscale reservoir system, which consists of macro-micro fracture system, micro intergranular pores and nanoscale organic intragranular pores. The sweet spots in the tight oil and gas reservoir are controlled by various factors. The endowment function of natural fracture in oil and gas exploration and development is of dual character: on the one hand, it changes the reservoir's seepage capability, improves the early production capacity of the tight oil and gas reservoir and contributes to a high gas production rate; On the other hand, the interaction between natural fracture development network of the reservoir and hydraulically created fracture is an important factor influencing the reservoir reformation volume, so as to influence the production capacity of producing well. Therefore, the existence of fracture plays a significant role in the exploration effect and hydrofracturing of tight oil and gas reservoir.
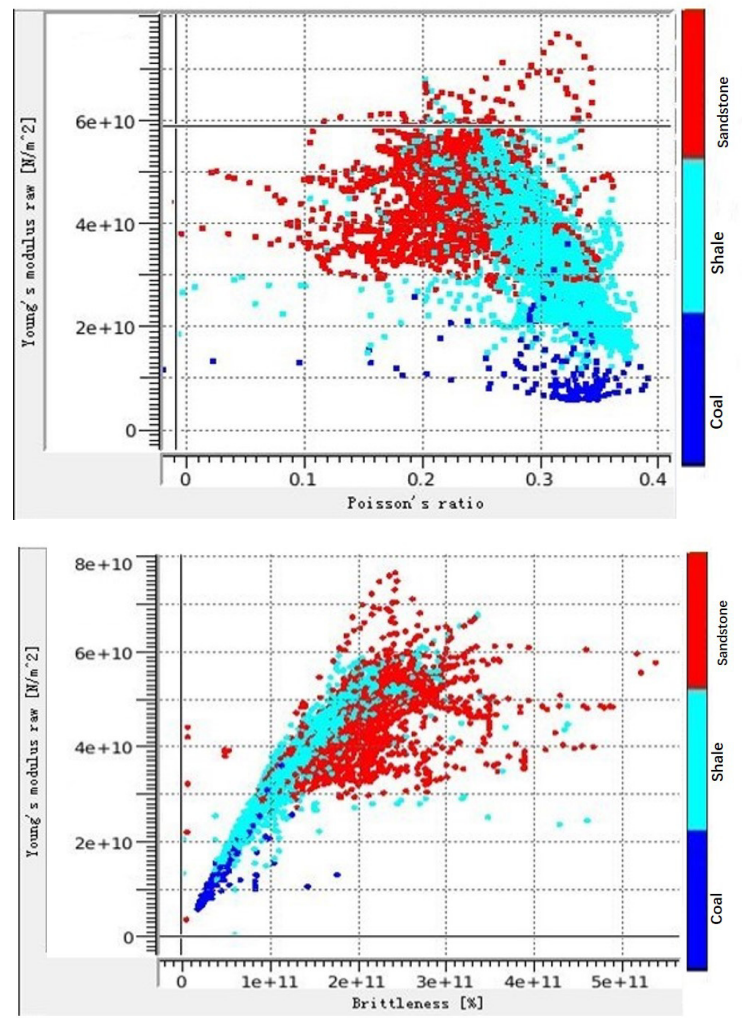

Fig. 4. Elastic parameter and brittleness index cross-plot.

\subsubsection{Fracture prediction under stress field constraint}

The theoretical basis for fracture prediction based on formation stress field analysis is structural mechanics. Starting from structural mechanics, the geometric information (structural plane) and lithological information (velocity and density) of the formation were used to estimate its stress field, curvature tensor, deformation tensor and stress field tensor of the formation plane, so as to obtain principal curvature, principal strain and principal stress. First, based on rock core and Formation MicroScanner Image (FMI) imaging data, single-well fracture identification and analysis were implemented. The fracture development characteristics and distribution laws were figured out and the fracture development intervals were clarified mainly through fracture formation mechanism, occurrence and length and filling degree analysis. Furthermore, the relationship between fracture and permeability at logging scale was established, and the qualitative and quantitative relationships between fracture development degree and FMI imaging data were determined, thus providing a foundation for the subsequent small-scale fracture prediction; The fracture belonging to natural earthquake level is usually a concomitant fracture nearby rupturing and folding belt caused by tectonic movement. The fracture arising out of non-tectonic movement cannot be identified. Multiscale and multi-method prediction and optimal selection are mainly carried out in the aspect of large-scale fracture prediction, and the main methods used include discontinuous detecting technique, curvature attribute-based fracture detecting technique, and ant tracking technique-based fracture detection method; In comparison with pre-stack seismic data prediction technique, pre-stack P-wave-assisted theoretical fracture detecting technique with positional anisotropism has higher sensitivity to meso and microscale fractures, but its limitations cannot be neglected, e.g. failing to effectively identify multigroup medium and low-angle fractures, being unable to identify fracture development phase or effectively characterize the relationship between fracture and rupture.

\subsection{Visualized fusion of "double sweet spots" data}

Fig. 5a shows the pre-stack fracture attribute fusion graph based on the coherence algorithm. It can be seen that the pre-stack fracture attribute of Member 4 of Shihezi Formation is quite developed in the northeast of Well A. From the planar graph of brittleness factor acquired through ore-stack inversion, the area with satisfying brittleness factor is located in the northeast of the work area. The brittleness factor value of Well A is also high, the reservoir area with advantaged effective reservoir thickness, which is obtained through inversion, is also developed in the northeast, and good reservoirs are developed in Well A, too. From the display image of "double sweet spots" attribute fusion of Member 4 of Shihezi Formation, the reservoir where Well A is located also has large thickness and brittleness values with low development degree of natural tectonic fracture, and this is to the advantage of reservoir fracturing reformation. High yield is obtained from Well A through the fracturing reformation (Fig. 5). 


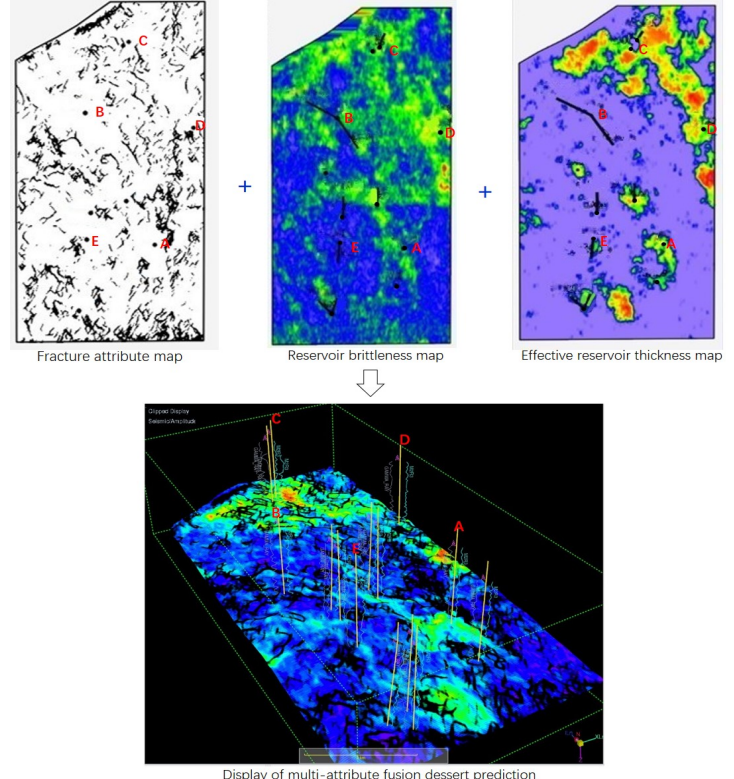

Fig. 5. "Double sweet spots" attribute fusion graph of Member 4 of Shihezi Formation.

\section{Conclusion}

(1) The petrophysical analysis indicates that the P-wave impedance has no evident identification window for a thin tight reservoir, but the VP/VS ratio does. The spatial distribution characteristics of reservoirs in the study area can be described through the VP/VS ratio data acquired through pre-stack and post-stack combined inversion;

(2) The selection of proper velocity field is of vital importance in the conversion process of offset gather into angle gather. The conversion angle is more stable in longitudinal direction at interval velocity than that at root mean square or average velocity. More real AVO response can be acquired by removing the gather;

(3) Engineering and mechanical parameters like brittleness factor are indispensable evaluation parameters in the prediction process of tight sandstone reservoirs. That engineering and mechanical parameters participate in the predictive evaluation of sweet spots in tight sandstone reservoir can mitigate its exploration and development risk;

(4) Differential stress field and differential stress field-based micro-seismic fracturing simulation have gradually become new research hotspots and difficulties in the comprehensive evaluation of tight sandstone reservoirs. Therefore, the applied research in this field remains to be further deepened.

\section{References}

1. Walls J D, Tight gas sands-permeability, pore structure and clay. J. Journal of Petroleum Technology, 34:2707-2714 (1982)

2. Law B E,Spencer W C, Gas in tight reservoirs:an emerging energy. J. USGS Professional Paper, 12(1):233-252 (1993)
3. Surdam R C, Boese S W, Crossey L J., The chemistry of secondary porosity. J. AAPG Memoir, 37 : 127-149 (1984)

4. Surdam R C, Crossey L J, Hagen E S., Organicinorganic and sandstone diagenesis. $\mathrm{J}$.AAPG Bulletin, 73:1-23 (1989)

5. Tong $\mathrm{X}$ G, Origin and distribution of unconventional oil. J. Journal of petroleum, S.1:20 26 (2012)

6. Zhu X M et al, Research progress and hot issues analysis of tight reservoir. Frontier of Geosciences,25(2):141-145 (2018)

7. ULIASZ-MISIAK B, PRZYBYCIN A, WINID B, Shale ang tight gas in Poland:legal environmental Issues. J. Energy policy, 65:68-77 (2014)

8. British Petroleum Company, BPstatitical review of world engery 2011. R. London:British Petroleum Company (2011)

9. XIA Z, LI ZC, JIA R Z, et al., Thin reservoir sedimentalry mocrofacies characterization based on well logging and seimic data:a case study. J. OIL Geophysical Prospecting, 5195:1002-1011 (2016)

10. GOODWAY B, PEREZM, VARSEKJ, et al., Seismic petrophysics and isotropic-anisotropic AVO methods for unconventional gas exploration. J. geophysics, 75(5):A65-A76 (2010) 